\title{
Marcus' electron transfer rate revisited via a Rice-Ramsperger-Kassel-Marcus analogue: A unified formalism for linear and nonlinear solvation scenarios
}

\author{
Yao Wang $*$ Yu Su, Rui-Xue $\mathrm{Xu}$, Xiao Zheng, and YiJing Yan \\ Hefei National Laboratory for Physical Sciences at the Microscale and Department of \\ Chemical Physics and Synergetic Innovation Center of Quantum Information and Quantum \\ Physics and Collaborative Innovation Center of Chemistry for Energy Materials (iChEM), \\ University of Science and Technology of China, Hefei, Anhui 230026, China
}

(Dated: January 5, 2021)

\begin{abstract}
In the pioneering work by R. A. Marcus, the solvation effect on electron transfer (ET) processes was investigated, giving rise to the celebrated nonadiabatic ET rate formula. In this work, on the basis of the thermodynamic solvation potentials analysis, we reexamine Marcus' formula with respect to the Rice-Ramsperger-Kassel-Marcus (RRKM) theory. Interestingly, the obtained RRKM analogue, which recovers the original Marcus' rate that is in a linear solvation scenario, is also applicable to the nonlinear solvation scenarios, where the multiple curve-crossing of solvation potentials exists. Parallelly, we revisit the corresponding Fermi's golden rule results, with some critical comments against the RRKM analogue proposed in this work. For illustration, we consider the quadratic solvation scenarios, on the basis of physically well-supported descriptors.
\end{abstract}

\section{INTRODUCTION}

Electron transfer (ET) is a fundamental and representative type of physical-chemistry processes. The Marcus' ET theory is considered to be a milestone for understanding the solvation effect in these processes. It gives rise to the celebrated nonadiabatic ET rate, which reads $1-4$

$$
k=\frac{V^{2} / \hbar}{\sqrt{\lambda k_{B} T / \pi}} \exp \left[-\frac{\left(E^{\circ}+\lambda\right)^{2}}{4 \lambda k_{B} T}\right] .
$$

Here, $V$ denotes the nonadiabatic transfer coupling parameter and $E^{\circ} \simeq \Delta G_{r}^{\circ}$ amounts to the standard reaction Gibbs energy, for the electron transferring from the donor $(|0\rangle$; reactant) to the acceptor $(|1\rangle$; product) state, with $\lambda$ being the associated solvent reorganization energy.

The minimum model for the total ET composite Hamiltonian assumes

$$
H_{\mathrm{ET}}=h_{0}|0\rangle\left\langle 0\left|+\left(E^{\circ}+h_{1}\right)\right| 1\right\rangle\langle 1|+V(|0\rangle\langle 1|+| 1\rangle\langle 0|) .
$$

The electronic system in donor and acceptor states are associated with their own solvent environments. The individual solvent environment is characterized by not only the Hamiltonian, $h_{a}$, but also $\rho_{a}^{\mathrm{E} ; \mathrm{eq}}(T) \equiv e^{-\beta h_{a}} / \operatorname{tr} e^{-\beta h_{a}}$, with $\beta=1 /\left(k_{B} T\right)$, where $k_{B}$ is the Boltzmann constant and $T$ the temperature. The total ET composite was initially $\rho_{\mathrm{T}}\left(t_{0}\right)=\rho_{0}^{\mathrm{E} ; \mathrm{eq}}(T)|0\rangle\langle 0|$, the thermal equilibrium in the donor state, prior to the nonadiabatic coupling $V$ taking action.

It is widely known that the Marcus' nonadiabatic ET rate formula, Eq. (1), can be derived via the Fermi's golden rule (FGR) in the linear solvation scenarios (cf. Sec.IA). However, in reality, some degrees of freedom that can be treated as a part of solvent environment are nonlinear ${ }^{5-12}$ Can the FGR approach be directly extended to nonlinear solvation scenarios? And why is this the case? To answer these questions, other perceptions of understanding Marcus' ET rate formula may be helpful.
To this end, it would be interesting to see whether the Marcus' ET rate formula can be reproduced from the prospect of Rice-Ramsperger-Kassel-Marcus (RRKM) theory $13-19$ This theory shares the common ground with the transition-state theory (TST) $20-22$ They both deal with the adiabatic events of first-passage over the barrier top where the activated complex is located. The underlying reaction kinetic mechanism is described with

$$
\mathrm{R} \stackrel{\text { pre-eq. }}{\rightleftharpoons} \mathrm{X}^{\ddagger} \stackrel{\text { slow }}{\longrightarrow} \mathrm{P}
$$

It starts with the fast pre-equilibrium step, forming activated complex $\mathrm{X}^{\ddagger}$, and then undergoes a slow "unimolecular dissociation" to the product $(\mathrm{P})$. For the latter step, from the activated complex to product, the TST describes with the Eyring's reactive coordinate frequency formula, ${ }^{21,22}$ whereas the RRKM theory goes by an ergodicity description, adopted originally by Hinshelwood $\stackrel{23}{ }$ Nevertheless, both TST and RRKM theory are considered to be the methods for adiabatic gasphase rate processes. Can the RRKM be applied to nonadiabatic condensed-phase ET rate processes?

In both TST and RRKM theory, the reactant $(\mathrm{R})$ involved in the first step of kinetic mechanism (3) consists of two gas molecules. What will be their correspondences in the condensed-phase ET rate processes? This issue could be addressed on the basis of the statistical quasi-particle description for the solvation effect on ET processes ${ }^{24-27}$ In other words, we visualize the solvation as a collective species. It collides with a solute molecule, forming an activated solute-solvent complex $\mathrm{X}^{\ddagger}$. This first step of the kinetic mechanism (3) is a fast pre-equilibrium process. The second step assumes a slow solvent relaxation, resulting in a stable ET product.

Based on the above ideas, in this work we propose a RRKM analogue that not only recovers the Marcus' nonadiabatic ET rate, Eq. (11), in the linear solvation scenario (cf. Sec. IIB), but also treats the nonlinear solvation scenarios, where the multiple curve-crossing of sol- 


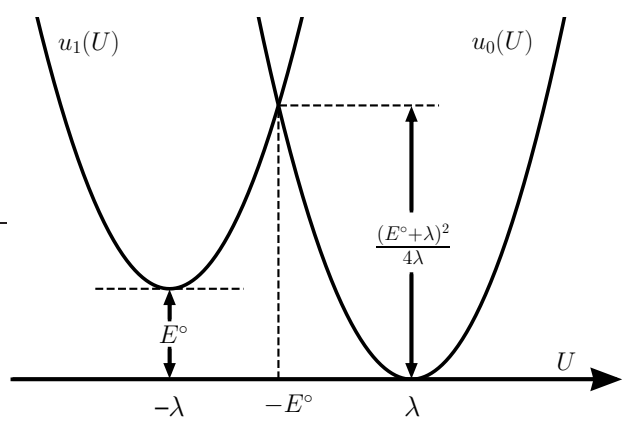

FIG. 1: The solvation potentials, $u_{0}(U)$ and $u_{1}(U)$ of Eq. (77). The curve-crossing, $u_{0}\left(U^{\ddagger}\right)=E^{\circ}+u_{1}\left(U^{\ddagger}\right)$, occurs at $U^{\ddagger}=$ $-E^{\circ}$. This determines the activation energy to be the value of $\Delta G^{\ddagger}=u_{0}\left(U^{\ddagger}\right)=\left(E^{\circ}+\lambda\right)^{2} /(4 \lambda)$.

vation potentials exists (cf. Sec.IIIB). Parallelly, we revisit the corresponding FGR results for comparisons (cf. Sec.IIIA).

As an illustration, we consider the quadratic solvation scenarios, on the basis of physically well-supported descriptors (cf. Sec.IV]) ${ }^{28-30}$ The results are discussed in different cases, with heuristic theoretical pictures. In Sec. $\mathrm{V}$ are concluding remarks, together with the prospect of this work.

\section{LINEAR SOLVATION SCENARIO}

In the linear solvation scenarios, the solvation coordinate $(X)$ is

$$
X=U \equiv h_{1}-h_{0}
$$

It is worth emphasizing beforehand that $X \neq U$ in the nonlinear solvation scenerios. The reorganization energy

$$
\lambda=\langle U\rangle_{0}=-\langle U\rangle_{1}>0
$$

where $\langle\hat{O}\rangle_{a} \equiv \operatorname{tr}\left[\hat{O} \rho_{a}^{\mathrm{E} ; \mathrm{eq}}(T)\right]$. One may elaborate the Marcus' ET theory with the solvation potentials $\left\{u_{\alpha}(U)\right\}$ that are thermodynamic measures. Each projects the microscopic solvent potentials $\left\{v_{\alpha}(\mathbf{x})\right\}$ onto the solvation coordinate as $\left\{u_{\alpha}(U)\right\}$, and $U=u_{1}(U)-u_{0}(U)$ is linear and one-dimensional. The resultant Boltzmann distribution proportional to $e^{-\beta u_{a}(U)}$ is a Gaussian function, with the mean value, Eq. (5), and the variance. The latter takes a common value as $U$ is linear. The classical hightemperature fluctuation-dissipation theorem ${ }^{31,32}$ gives rise to the Gaussian variance

$$
\left\langle\delta U^{2}\right\rangle_{0}=\left\langle U^{2}\right\rangle_{0}-\langle U\rangle_{0}^{2} \approx 2 \lambda k_{B} T .
$$

It together with Eq. (5) leads to

$$
u_{0}(U)=\frac{1}{4 \lambda}(U-\lambda)^{2} \quad \text { and } \quad u_{1}(U)=\frac{1}{4 \lambda}(U+\lambda)^{2} .
$$

Figure11depicts these solvation potentials. The resultant activation energy $\Delta G^{\ddagger}$ via the curve-crossing condition agrees perfectly with that in Eq. (11).

\section{A. FGR elaboration}

Let us start with the FGR derivations. The universal FGR rate formula reads

$$
k=\frac{2 V^{2}}{\hbar^{2}} \operatorname{Re} \int_{0}^{\infty} \mathrm{d} t e^{-\frac{i}{\hbar} E^{\circ} t}\left\langle\exp _{+}\left[-\frac{i}{\hbar} \int_{0}^{t} \mathrm{~d} \tau U(\tau)\right]\right\rangle .
$$

Here, $U(t) \equiv e^{i h_{0} t / \hbar} U e^{-i h_{0} t / \hbar}$ and the the ensemble average, $\langle\cdot\rangle$, runs over the initial $\rho_{0}(U)=e^{-\beta u_{0}(U)} / \mathcal{Z}$. Here, the partition function

$$
\mathcal{Z} \equiv \int_{-\infty}^{\infty} e^{-\beta u_{0}(U)} \mathrm{U}=\sqrt{4 \pi \lambda k_{B} T}
$$

In the static limit, $U(\tau) \approx U \equiv u_{1}(X)-u_{0}(X)$, one can then perform the time integration first, resulting in Eq. (8) the expression,

$$
k=\frac{2 \pi V^{2}}{\hbar}\left\langle\delta\left[u_{1}(U)-u_{0}(U)+E^{\circ}\right]\right\rangle .
$$

This together with $\rho_{0}(U)=e^{-\beta u_{0}(U)} / \sqrt{4 \pi \lambda k_{B} T}$ recovers the Marcus' ET rate in Eq. (11).

\section{B. RRKM analogue}

Presented above is the FGR elaboration. Now turn to the RRKM analogue to derive Eq. (11). In analogy to the RRKM theory, we apply the ergodicity description. 33 Consider a ET system of total energy $E$ at the donor state $|0\rangle$, and therefore

$$
E=\frac{1}{2} m_{\mathrm{eff}} \dot{U}^{2}+u_{0}(U)
$$

The first term in Eq. (11) represents the kinetic energy, where $\dot{U} \equiv \mathrm{U} / \mathrm{t}$ is the moving velocity of the solvation coordinate and $m_{\text {eff }}$ is the effective mass of the solvation collective species to be identified below. In the nonadiabatic ET pictures, the reaction rate

$$
k(E)=\nu(E) P_{0 \rightarrow 1}(E) .
$$

Here, $\nu(E)$ is the frequency of the system arriving at the crosspoint $U^{\ddagger}$, where the "activated complex" is located. It is twice as large as the temporal frequency (angular frequency divided by $2 \pi$ ) reading

$$
\nu(E)=2 \cdot(\omega / 2 \pi),
$$

where $\omega$ is the angular frequency of the ET system at the donor state, and the factor 2 is due to the fact that the system arrives at the crosspoint twice in each period. In Eq. (12), $P_{0 \rightarrow 1}(E)$ is the probability of the electron transferring from $|0\rangle$ to $|1\rangle$ at the crosspoint of two adiabatic potential surfaces $u_{0}(U)$ and $u_{1}(U)$. It adopts the Landau-Zener (LZ) form as ${ }^{34-36}$

$$
P_{0 \rightarrow 1}(E)=1-\exp \left[-\frac{2 \pi V^{2} / \hbar}{\left|u_{0}^{\prime}\left(U^{\ddagger}\right)-u_{1}^{\prime}\left(U^{\ddagger}\right)\right|\left|\dot{U}^{\ddagger}\right|}\right] .
$$


In Eq. (14), $u^{\prime}\left(U^{\ddagger}\right) \equiv u ̛ /\left.U\right|_{U=U^{\ddagger}}$ is the slope of potential surface at the crosspoint, and the velocity $\left|\dot{U}^{\ddagger}\right|=$ $\left[2\left(E-\Delta G^{\ddagger}\right) / m_{\mathrm{eff}}\right]^{\frac{1}{2}}$ according to Eq. (11). Furthermore, according to the Fig.1] at the crosspoint $U^{\ddagger}=-E^{\circ}[\mathrm{cf}$. Eq. (7)],

$$
\left|u_{0}^{\prime}\left(U^{\ddagger}\right)-u_{1}^{\prime}\left(U^{\ddagger}\right)\right|=1 .
$$

These give rise to

$$
P_{0 \rightarrow 1}(E)=1-\exp \left[-\frac{2 \pi V^{2} / \hbar}{\left[2\left(E-\Delta G^{\ddagger}\right) / m_{\mathrm{eff}}\right]^{\frac{1}{2}}}\right] .
$$

In the weak coupling regimes, Eq. (16) can be approximated as

$$
P_{0 \rightarrow 1}(E) \simeq \frac{2 \pi V^{2} / \hbar}{\left[2\left(E-\Delta G^{\ddagger}\right) / m_{\mathrm{eff}}\right]^{\frac{1}{2}}},
$$

and then we can obtain

$$
k(E)=\nu(E) P_{0 \rightarrow 1}(E)=\frac{\left(2 \omega^{2} m_{\mathrm{eff}}\right)^{\frac{1}{2}} V^{2} / \hbar}{\left(E-\Delta G^{\ddagger}\right)^{\frac{1}{2}}} .
$$

Similar to the RRKM theory, the overall rate of ET at temperature $T$ reads

$$
k=\int_{\Delta G^{\ddagger}}^{\infty} \mathrm{d} E k(E) \frac{e^{-\beta E}}{Z},
$$

with the partition function

$$
Z \equiv \int_{0}^{\infty} \mathrm{d} E e^{-\beta E}=k_{B} T .
$$

After some simple algebra, we obtain from Eq. (19) that

$$
k=\frac{\left(2 \omega^{2} m_{\mathrm{eff}}\right)^{\frac{1}{2}} V^{2} / \hbar}{\sqrt{k_{B} T / \pi}} \exp \left[-\frac{\left(E^{\circ}+\lambda\right)^{2}}{4 \lambda k_{B} T}\right] .
$$

Compare with the Marcus' rate in Eq. (11), we know that

$$
2 \omega^{2} m_{\mathrm{eff}}=1 / \lambda
$$

This fact is also verified via the detailed analysis of the quadratic solvation scenarios in Sec.IVA [cf. Eq. (61) with $\theta=1]$. We then recover the Marcus' nonadiabatic ET rate formula, Eq. (11), via this RRKM analogue.

In the following section, according to this RRKM analogue, we will construct a generalized rate formula for ET processes in the nonlinear solvation scenarios, where the multiple curve-crossing of solvation potentials exists.

\section{NONLINEAR SOLVATION SCENARIO}

In general, the energy difference, $U=h_{1}-h_{0}$, defined in Eq. (44) is nonlinear, i.e., $X \neq U$, with $X$ up to the linear part of $U$. Therefore, the thermodynamic solvation potentials hereafter are $u_{0}(X)$ and $u_{1}(X)$, instead of $u_{0}(U)$ and $u_{1}(U)$, associated with the reactant and product, respectively. In the nonlinear solvation scenarios, there may exist multiple curve-crossing of solvation potentials, resulting in multiple crosspoints. Let $X_{n}^{\ddagger}$ be the $n$th crossing point, with the barrier height, $\Delta G_{n}^{\ddagger}$, satisfying

$$
\Delta G_{n}^{\ddagger}=u_{0}\left(X_{n}^{\ddagger}\right)=u_{1}\left(X_{n}^{\ddagger}\right)+E^{\circ} .
$$

In the remaining part of this section, we elaborate the ET rate formula via both the FGR and the RRKM analogue.

\section{A. FGR elaboration}

Similar to Eq. (10), the FGR rate in this scenario reads

$$
k=\frac{2 \pi V^{2}}{\hbar}\left\langle\delta\left[u_{1}(X)-u_{0}(X)+E^{\circ}\right]\right\rangle
$$

with the ensemble average, $\langle\cdot\rangle$, runs over the initial state $\rho_{0}(X)=e^{-\beta u_{0}(X)} / \mathcal{Z}$ where

$$
\mathcal{Z} \equiv \int_{-\infty}^{\infty} e^{-\beta u_{0}(X)} \mathrm{X}
$$

Mathematically, the evaluation is carried out with the roots of involving delta function by solving $u_{1}(X)-$ $u_{0}(X)+E^{\circ}=0$. The solutions just satisfy the second expression of Eq. (23). Now by applying the identity,

$$
\int_{-\infty}^{\infty} \mathrm{d} x f(x) \delta[g(x)]=\sum_{i} \frac{f\left(x_{i}\right)}{\left|g^{\prime}\left(x_{i}\right)\right|},
$$

where $g^{\prime}\left(x_{i}\right)$ denotes the slope of $g(x)$ at the root $x=x_{i}$. Note that $f\left(x_{i}\right)=0$ if $x_{i}$ is not a real root and $f(x)$ is a real distribution function, such as $\rho_{0}(X)$ in the present study. The resultant rate reads

$$
k=\frac{2 \pi V^{2} / \hbar}{\mathcal{Z}} \sum_{n} \frac{1}{\left|u_{0}^{\prime}\left(X_{n}^{\ddagger}\right)-u_{1}^{\prime}\left(X_{n}^{\ddagger}\right)\right|} \exp \left(-\frac{\Delta G_{n}^{\ddagger}}{k_{B} T}\right) .
$$

There are two limiting scenarios, as follows. One is the Marcus' linear solvation scenario, with Eq. (77) being just $u_{0}(X)=(X-\lambda)^{2} /(4 \lambda)$ and $u_{1}(X)=(X+\lambda)^{2} /(4 \lambda)$. In this case, $\left|u_{0}^{\prime}(X)-u_{1}^{\prime}(X)\right|=1$. It is easy to verify that all the results in Sec.II are reproduced. Another limiting scenario is $u_{0}^{\prime}\left(X^{\ddagger}\right)=u_{1}^{\prime}\left(X^{\ddagger}\right)$. This is the case that two potential curves are just barely touched. In this critical case, Eq. (27) breaks down due to the divergence. From the perception of RRKM analogue elaborated in Sec.IIB. we know that it is invalid to do the approximation in Eq. (17) when $\left|u_{0}^{\prime}(X)-u_{1}^{\prime}(X)\right|$ is relatively small. It leads to the divergence in this limiting case.

\section{B. RRKM analogue}

Due to the breakdown of approximation in Eq. (17), we directly apply the original LZ form of transition probability. Similarly, consider a ET system of total energy $E$ 
at the donor state $|0\rangle$, and therefore

$$
E=\frac{1}{2} m_{\mathrm{eff}} \dot{X}^{2}+u_{0}(X)
$$

The reaction rate associated with $E$ through $n$th crosspoint is then

$$
k_{n}(E)=\nu_{n}(E) P_{0 \rightarrow 1 ; n}(E) .
$$

Similarly,

$$
\nu_{n}(E)=2 \cdot(\omega / 2 \pi),
$$

and the LZ form of transition probability reads

$$
P_{0 \rightarrow 1 ; n}(E)=1-\exp \left[-\frac{2 \pi V^{2} / \hbar}{\left|u_{0}^{\prime}\left(X_{n}^{\ddagger}\right)-u_{1}^{\prime}\left(X_{n}^{\ddagger}\right)\right|\left|\dot{X}_{n}^{\ddagger}\right|}\right],
$$

with $\left|\dot{X}_{n}^{\ddagger}\right|=\left[2\left(E-\Delta G_{n}^{\ddagger}\right) / m_{\text {eff }}\right]^{\frac{1}{2}}$. Then the overall rate of ET at temperature $T$ reads

$$
k=\sum_{n} \int_{\Delta G_{n}^{\ddagger}}^{\infty} \mathrm{d} E k_{n}(E) \frac{e^{-\beta E}}{Z},
$$

with $Z=k_{B} T$. Here, the sum runs over all the reactive channels to the product. We then obtain

$$
k=\frac{\omega}{\pi k_{B} T} \sum_{n} \int_{\Delta G_{n}^{\ddagger}}^{\infty} \mathrm{d} E P_{0 \rightarrow 1 ; n}(E) e^{-\beta E},
$$

with

$P_{0 \rightarrow 1 ; n}(E)=1-\exp \left[-\frac{\left(2 m_{\mathrm{eff}}\right)^{\frac{1}{2}} \pi V^{2} / \hbar}{\left|u_{0}^{\prime}\left(X_{n}^{\ddagger}\right)-u_{1}^{\prime}\left(X_{n}^{\ddagger}\right)\right|\left(E-\Delta G_{n}^{\ddagger}\right)^{\frac{1}{2}}}\right]$.

Use the formula

$$
\int_{0}^{\infty} \mathrm{d} t t e^{-2 a / t-b t^{2}}=\frac{a^{2}}{2 \sqrt{\pi}} G_{0,0}^{3,0}\left(\left.a^{2} b\right|_{-1,-1 / 2,0}\right),
$$

where $G_{0,0}^{3,0}\left(\left.x\right|_{-1,-1 / 2,0}\right)$ is the corresponding Meijer Gfunction, and we can obtain

$$
k=\frac{\omega}{\pi} \sum_{n} \eta_{n} \exp \left(-\frac{\Delta G_{n}^{\ddagger}}{k_{B} T}\right)
$$

with

$$
\eta_{n}=1-\frac{\kappa_{n}^{2} G_{0,0}^{3,0}\left(\left.\beta \kappa_{n}^{2}\right|_{-1,-1 / 2,0}\right)}{\sqrt{\pi} k_{B} T}
$$

where

$$
\kappa_{n}=\frac{\left(m_{\mathrm{eff}} / 2\right)^{\frac{1}{2}} \pi V^{2} / \hbar}{\left|u_{0}^{\prime}\left(X_{n}^{\ddagger}\right)-u_{1}^{\prime}\left(X_{n}^{\ddagger}\right)\right|} .
$$

Now consider the limiting scenario where $u_{0}^{\prime}\left(X^{\ddagger}\right)=$ $u_{1}^{\prime}\left(X^{\ddagger}\right)$. We have $\eta_{n} \rightarrow 1$, and the corresponding rate formula in Eq. (36) does not suffer the divergence any more. In this rate formula, the angular frequency $\omega$ and the effective mass $m_{\text {eff }}$ are to be determined with respect to concrete settings. In the next section, as an illustrative example, we will consider the quadratic solvation scenarios, on the basis of physically well-supported descriptors $\stackrel{28-30}{2}$

\section{ELECTRON TRANSFER IN QUADRATIC SOLVATION ENVIRONMENTS}

\section{A. Quadratic thermodynamic potentials analysis}

It is worth emphasizing that a physical description on the system-environment couplings in solutions should satisfy the invariance requirement, with respect to the reference environment. For a linear coupling solvation environment, this requirement is automatically satisfied. However, it is a nontrivial task even for quadratic solvation environments ${ }^{28-30}$ Presented below is the recently developed quadratic solvation descriptors scheme ${ }^{29}$ that satisfies the aforementioned reference-environment invariance requirement.

Recall that $h_{0}$ and $h_{1}$ are the environment Hamiltonians associated with the electronic system in donor and acceptor states, respectively [cf. Eq. (2)]. In contact to the solvation modes description, we express these two environment Hamiltonian in the Calderia-Leggett's form,

$$
\begin{aligned}
& h_{0}=\frac{\omega}{2}\left(\hat{p}^{2}+\hat{x}^{2}\right)+\sum_{k} \frac{\tilde{\omega}_{k}}{2}\left[\tilde{p}_{k}^{2}+\left(\tilde{x}_{k}-\frac{\tilde{c}_{k}}{\tilde{\omega}_{k}} \hat{x}\right)^{2}\right], \\
& h_{1}=\frac{\omega^{\prime}}{2}\left(\hat{p}^{\prime 2}+\hat{x}^{\prime 2}\right)+\sum_{k} \frac{\tilde{\omega}_{k}^{\prime}}{2}\left[\tilde{p}_{k}^{\prime 2}+\left(\tilde{x}_{k}^{\prime}-\frac{\tilde{c}_{k}^{\prime}}{\tilde{\omega}_{k}^{\prime}} \hat{x}^{\prime}\right)^{2}\right] .
\end{aligned}
$$

Physically, each expression describes a Brownian oscillator (first term), under the influence of secondary environment. One can readily obtain the generalized Langevin equation, in which the friction kernel reads 32

$$
\begin{aligned}
\zeta(t) & =\omega \sum_{k} \frac{\tilde{c}_{k}^{2}}{\tilde{\omega}_{k}} \cos \left(\tilde{\omega}_{k} t\right), \\
\zeta^{\prime}(t) & =\omega^{\prime} \sum_{k} \frac{\tilde{c}_{k}^{\prime 2}}{\tilde{\omega}_{k}^{\prime}} \cos \left(\tilde{\omega}_{k}^{\prime} t\right) .
\end{aligned}
$$

The $h_{1}$-environment is subject to linear-displacements and frequency-shifts in relation to $h_{0}$. This can be described as

$$
\begin{aligned}
& \hat{x}^{\prime}=\left(\omega^{\prime} / \omega\right)^{\frac{1}{2}}(\hat{x}-d) \equiv \theta^{\frac{1}{2}}(\hat{x}-d), \\
& \tilde{x}_{k}^{\prime}=\left(\tilde{\omega}_{k}^{\prime} / \tilde{\omega}_{k}\right)^{\frac{1}{2}}\left(\tilde{x}_{k}-\tilde{d}_{k}\right) \equiv \tilde{\theta}_{k}^{\frac{1}{2}}\left(\tilde{x}_{k}-\tilde{d}_{k}\right) .
\end{aligned}
$$

The kinetics energies in $h_{0}$ and $h_{1}$ are the same. Similar to Eq. (4), the solvation energy with respect to each individual reference environment is given respectively by

$$
\begin{aligned}
& \delta h_{0} \equiv h_{1}-h_{0}=\alpha_{0}+\alpha_{1} \hat{x}+\alpha_{2} \hat{x}^{2}, \\
& \delta h_{1} \equiv h_{0}-h_{1}=\alpha_{0}^{\prime}+\alpha_{1}^{\prime} \hat{x}^{\prime}+\alpha_{2}^{\prime} \hat{x}^{\prime 2} .
\end{aligned}
$$

The involving descriptors $\left\{\alpha_{i}\right\}$ and $\left\{\alpha_{i}^{\prime}\right\}$ should satisfy the reference-environment invariance that reads

$$
\begin{aligned}
& h_{0}+\hat{Q}_{\mathrm{S}}\left(\alpha_{0}+\alpha_{1} \hat{x}+\alpha_{2} \hat{x}^{2}\right) \\
= & h_{1}+\left(1-\hat{Q}_{\mathrm{S}}\right)\left(\alpha_{0}^{\prime}+\alpha_{1}^{\prime} \hat{x}^{\prime}+\alpha_{2}^{\prime} \hat{x}^{\prime 2}\right) .
\end{aligned}
$$


Here $\hat{Q}_{\mathrm{S}}$ describes the dissipative system mode on which the environment acts. In contact with the ET system, Eq. (2), $\hat{Q}_{\mathrm{S}}=|1\rangle\langle 1|$. To determine the descriptors, we adopt the linear-displacement-mapping method, which results in 29

$$
\lambda \equiv\langle\delta h\rangle_{\alpha_{2}=0}=\frac{1}{2} \omega d^{2}
$$

and

$$
\alpha_{0}=\lambda \theta^{2}, \quad \alpha_{1}=-(2 \lambda \omega)^{\frac{1}{2}} \theta^{2}, \quad \alpha_{2}=\frac{\omega}{2}\left(\theta^{2}-1\right),
$$

whereas

$$
\alpha_{0}^{\prime}=\lambda, \quad \alpha_{1}^{\prime}=(2 \lambda \omega / \theta)^{\frac{1}{2}}, \quad \alpha_{2}^{\prime}=\frac{\omega}{2 \theta}\left(1-\theta^{2}\right) .
$$

The derivations were made in the constraint of the environment-reference invariance, Eq. (43). Moreover, the linear displacement mapping ansatz implies also 29

$$
\zeta(t)=\zeta^{\prime}(t)
$$

To obtain the thermodynamic potentials, let us start with $v_{0}(\hat{\mathbf{x}})$ and $v_{1}\left(\hat{\mathbf{x}}^{\prime}\right)$, the potential energies of $h_{0}$ and $h_{1}$, respectively [cf. Eq. (39)]. Project them to the same solvation coordinate $\hat{x}$, resulting in the thermodynamic potentials, $u_{0}(\hat{x})$ and $u_{1}(\hat{x})$. By doing this, we can recast Eq. (42a) as

$$
U=u_{1}(\hat{x})-u_{0}(\hat{x})=\alpha_{0}+\alpha_{1} \hat{x}+\alpha_{2} \hat{x}^{2} .
$$

To facilitate the analysis in line with Eq. (7) and Fig.11 we introduce

$$
\begin{gathered}
X \equiv \alpha_{0}+\alpha_{1} \hat{x} \\
X^{\prime} \equiv \alpha_{0}^{\prime}+\alpha_{1}^{\prime} \hat{x}^{\prime} .
\end{gathered}
$$

The thermodynamic potentials associated with $h_{0}$ and $h_{1}$ would read

$$
\begin{aligned}
u_{0}(X) & =\frac{1}{4 \Theta_{0} \lambda_{0}}\left(X-\lambda_{0}\right)^{2}, \\
u_{1}\left(X^{\prime}\right) & =\frac{1}{4 \Theta_{1} \lambda_{1}}\left(X^{\prime}-\lambda_{1}\right)^{2} .
\end{aligned}
$$

The parameters $\lambda_{0}, \lambda_{1}, \Theta_{0}$ and $\Theta_{1}$ are to be determined. Firstly, the definitions in Eq. (49) tells us

$$
\langle X\rangle_{0}=\alpha_{0} \quad \text { and } \quad\left\langle X^{\prime}\right\rangle_{1}=\alpha_{0}^{\prime}
$$

and therefore,

$$
\lambda_{0}=\alpha_{0}=\lambda \theta^{2} \quad \text { and } \quad \lambda_{1}=\alpha_{0}^{\prime}=\lambda .
$$

To proceed, we need to relate the primed quantities to the unprimed counterparts. By using Eqs. (41a), 44) and (49a), we obtain

$$
X^{\prime}=\left[\alpha_{0}^{\prime}-\alpha_{1}^{\prime}(2 \lambda \theta / \omega)^{\frac{1}{2}}\right]+\alpha_{1}^{\prime} \theta^{\frac{1}{2}}\left(X-\alpha_{0}\right) / \alpha_{1} .
$$

Here, $\alpha_{0}^{\prime}=\theta^{-2} \alpha_{0}, \alpha_{1}^{\prime}=-\theta^{-5 / 2} \alpha_{1}$ and $\alpha_{0} / \alpha_{1}=-d / 2$, as inferred from Eq. (46) versus Eq. (45). Therefore, $X^{\prime}=$ $-X / \theta^{2}$, which leads to Eq. (50) the expression,

$$
\begin{aligned}
& u_{0}(X)=\frac{1}{4 \Theta_{0} \lambda \theta^{2}}\left(X-\lambda \theta^{2}\right)^{2}, \\
& u_{1}(X)=\frac{1}{4 \Theta_{1} \lambda \theta^{4}}\left(X+\lambda \theta^{2}\right)^{2} .
\end{aligned}
$$

Together with Eq. (49a), we obtain

$$
\begin{aligned}
u_{1}-u_{0}= & \frac{\left(\alpha_{0}+\alpha_{1} \hat{x}+\lambda \theta^{2}\right)^{2}}{4 \Theta_{1} \lambda \theta^{4}} \\
& -\frac{\left(\alpha_{0}+\alpha_{1} \hat{x}-\lambda \theta^{2}\right)^{2}}{4 \Theta_{0} \lambda \theta^{2}} .
\end{aligned}
$$

By comparing with Eq. (48), we have also

$$
\Theta_{0}=\Theta_{1}^{-1}=\theta^{2},
$$

which further leads to Eq. (54) the expression,

$$
\begin{aligned}
& u_{0}(X)=\frac{1}{4 \lambda \theta^{4}}\left(X-\lambda \theta^{2}\right)^{2}, \\
& u_{1}(X)=\frac{1}{4 \lambda \theta^{2}}\left(X+\lambda \theta^{2}\right)^{2} .
\end{aligned}
$$

In Eq. (57) are the thermodynamic solvation potentials to be used later.

Now turn to the kinetic energy term to obtain the effective mass $m_{\mathrm{eff}}$. In $h_{0}$, according to Eqs. (39) and (49a), we can recast the kinetic energy term as

$$
\frac{1}{2} \omega \hat{p}^{2}=\frac{1}{2} \omega \alpha_{1}^{2} P^{2}
$$

where $[\hat{x}, \hat{p}]=[X, P]=i$. Therefore, the canonical equation of motion gives

$$
\dot{X}=\omega \alpha_{1}^{2} P
$$

The kinetic energy term can be then expressed as

$$
\frac{1}{2} \omega \alpha_{1}^{2} P^{2}=\frac{1}{2} m_{\mathrm{eff}} \dot{X}^{2},
$$

with

$$
m_{\mathrm{eff}}=\frac{1}{\omega \alpha_{1}^{2}}=\frac{1}{2 \lambda \omega^{2} \theta^{4}} .
$$

This recovers Eq. (22) in Sec. [B when $\theta=1$.

\section{B. ET rate in quadratic solvation environments}

In Sec.IVA we retain Eq. (4) as the general definition of $U$, and Eq. (5) for $\lambda$ is no longer valid, whenever $\theta \neq 1$. Note also that $\theta>0$, as implied in Eq. (46). Apparently, Eq. (57a) leads to Eq. (25) the value

$$
\mathcal{Z}=\left(4 \pi \lambda k_{B} T\right)^{\frac{1}{2}} \theta^{2}
$$


(a) double crossing

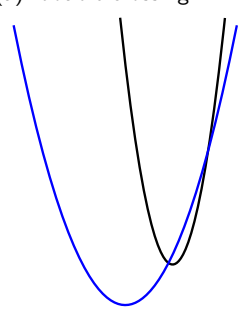

(b) no crossing

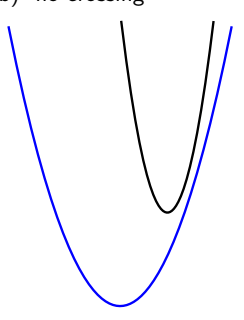

(c) critical

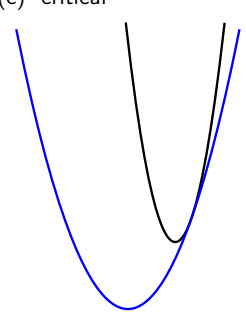

FIG. 2: Three representing scenarios, in relation to the curvecrossing behaviors, involving two solvation potentials with different curvatures $(\theta \neq 1)$. (a) Double crossing case; (b) No crossing case; (c) Critical case: $u_{0}^{\prime}\left(X^{\ddagger}\right)=u_{1}^{\prime}\left(X^{\ddagger}\right)$. The potential surfaces are plotted as the functions of $X$ [cf. Eq. (57)], and $X \neq U$ in this quadratic solvation scenarios [cf. Eqs. (48) and [49)].

According to Eq. (57), shown in Fig.2 are three representing scenarios, in relation to the curve-crossing behaviors, involving two solvation potentials with different curvatures $(\theta \neq 1)$. As discussed earlier, Fig.2(a) represents the multiple curve-crossing case, with the ET rate $k>0$, whereas (b) goes with $k=0$, as there are no crossing points. Figure 2(c) represents the critical scenario of $u_{0}^{\prime}\left(X^{\ddagger}\right)=u_{1}^{\prime}\left(X^{\ddagger}\right)$.

As seen below, one can define the quadratic characteristic parameter,

$$
q_{c} \equiv \frac{\left(1-\theta^{2}\right) E^{\circ}}{\theta^{2} \lambda} .
$$

The three representing scenarios of Fig. 2 are related to (a) $1+q_{c}>0$, (b) $<0$ and (c) $=0$, respectively.

As the kinetic rate process is concerned, in the following we focus only on the case of Fig.2(a). For the specified solvation potentials, Eq. (57), we obtain Eq. (23) the solutions,

$$
X_{ \pm}^{\ddagger}=-E^{\circ}+\Lambda_{ \pm}
$$

with [cf. Eq. [63)]

$$
\Lambda_{ \pm}=\frac{\lambda \theta^{2}}{1-\theta^{2}}\left(\sqrt{\left|1+q_{c}\right|} \pm \theta\right)^{2} .
$$

The corresponding activation energy reads

$$
\Delta G_{ \pm}^{\ddagger}=u_{0}\left(X_{ \pm}\right)=\frac{1}{4 \lambda \theta^{4}}\left(E^{\circ}+\lambda \theta^{2}-\Lambda_{ \pm}\right)^{2} .
$$

The resultant FGR rate acquires the form of Eq. (27), which reads

$$
k=\frac{2 \pi V^{2} / \hbar}{\mathcal{Z}} \eta^{\mathrm{FGR}} \sum_{ \pm} \exp \left(-\frac{\Delta G_{ \pm}^{\ddagger}}{k_{B} T}\right) .
$$

with

$$
\eta^{\mathrm{FGR}}=\frac{\theta}{\sqrt{\left|1+q_{c}\right|}}
$$

In the linear solvation case $(\theta=1), q_{c}=0$ via Eq. (63), resulting in the value of 1 for Eq. (68). However, when $1+q_{c} \rightarrow 0, \eta^{\mathrm{FGR}} \rightarrow \infty$, suffering a divergence. This is the scenario depicted in Fig.2(2), where the slopes at the pseudo-crossing point are of $u_{0}^{\prime}\left(X^{\ddagger}\right)=u_{1}^{\prime}\left(X^{\ddagger}\right)$. In this limiting case, we must consult the RRKM analogue. Eq. (36) tells us

$$
k=\frac{\omega}{\pi} \exp \left[-\frac{\lambda \theta^{4}}{k_{B} T\left(1-\theta^{2}\right)^{2}}\right]
$$

in this limiting case, where $\omega$ is as defined in Eq. (39).

\section{CONCLUDING REMARKS}

In summary, we have proposed a RRKM analogue to ET processes. Not only does it recover the original Marcus' rate, the proposed theory is also applicable to the nonlinear solvation scenarios, where multiple curvecrossing of solvation potentials exists. It is noticed that the original RRKM theory is concerned only with adiabatic gas-phase reactions $\underline{13}-19$ We elaborate this widely used kinetic mechanism from the analogous aspect, as detailed in Sec.IIB and Sec.IIB The obtained RRKM analogue is constructed on the basis of the ergodicity description, where we have also used the LZ formula for the transition probability.

We also revisit the corresponding FRG formula, with some critical comments against the RRKM analogue proposed in this work. Both approaches result in the Arrhenius-type expression of rate, with same activation energies in exponentials, but distinct pre-exponential coefficients. We scrutinize their differences particularly in the scenario of Fig.2(c), as highlighted at the end of Sec.IVB While the new theory gives the rate a finite value in this scenario, the FGR would result in $k \rightarrow \infty$. This also highlights the critical importance of an appropriate pre-exponential factor, in particular when the curve-crossing is degenerate.

However, we notice there still exists a discontinuity of the ET rate between the critical case $(k \neq 0)$ and the no crossing case $(k=0)$. To tackle with this discontinuity, we may further consult the Rosen-Zener nonadiabatic transition probability for the no crossing cases. As introduced in the literature, $\stackrel{38}{=}$ we may adopt

$$
P_{0 \rightarrow 1}(E)=1-\frac{\sinh \left[\left(D^{2}-1\right) \Gamma\right]}{\sinh \left(D^{2} \Gamma\right)} e^{-\Gamma}
$$

in place of Eq. (31). In Eq. (70),

$$
\Gamma=\frac{2 \pi V^{2} / \hbar}{\left|u_{0}^{\prime}\left(X^{\ddagger}\right)-u_{1}^{\prime}\left(X^{\ddagger}\right)\right|\left|\dot{X}^{\ddagger}\right|},
$$

and

$$
D=\left\{1+\frac{4 V^{2}}{\left[u_{0}\left(X^{\ddagger}\right)-u_{1}\left(X^{\ddagger}\right)-E^{\circ}\right]^{2}}\right\}^{\frac{1}{2}} .
$$


When there is no crossing, $X^{\ddagger}$ denotes the real part of the complex solutions of the equation $u_{0}(X)=u_{1}(X)+E^{\circ}$, which leads to $u_{0}\left(X^{\ddagger}\right)-u_{1}\left(X^{\ddagger}\right)-E^{\circ} \neq 0$. However, in the crossing cases, $u_{0}\left(X^{\ddagger}\right)-u_{1}\left(X^{\ddagger}\right)-E^{\circ}=0$, which results in $D \rightarrow \infty$ and therefore $\sinh \left[\left(D^{2}-1\right) \Gamma\right] / \sinh \left(D^{2} \Gamma\right) \rightarrow 1$. This gives back to the LZ formula, and all results in Sec.IIIB are reproduced. Consequently, this modification would eliminate the existing discontinuity of the ET rate between the critical case and the no crossing case. It not only revisits the RRKM analogue results in the crossing and critical cases, but also leads to $k \neq 0$ in the no crossing cases.

It is also worth noting that the present work treats the solvent environments in the static and classical limit. The failure of FGR treatment in the critical case [cf. Fig.2(c)] may imply that the dynamical fluctuation is an intrinsic nature associated with the nonlinearity. Fur- thermore, in the quantum regime, the nonlinear effects may become more prominent $28-30$ The chemical kinetics based on microscopic descriptions, together with exact quantum dissipative dynamics, may help further understanding the dynamical nonlinear effects in the ET rate processes in this regime.

\section{Acknowledgments}

Support from the Ministry of Science and Technology of China (Nos. 2017YFA0204904 \& 2016YFA0400904), the Natural Science Foundation of China (Nos. 21633006) and Anhui Initiative in Quantum Information Technologies is gratefully acknowledged.
* Electronic address: wy2010@ustc.edu.cn

1 R. A. Marcus, J. Chem. Phys. 24, 966 (1956).

${ }^{2}$ R. A. Marcus, Annu. Rev. Phys. Chem. 15, 155 (1964).

3 H. Sumi and R. A. Marcus, J. Phys. Chem. 84-9, 4894 (1986).

4 R. A. Marcus, Rev. Mod. Phys. 65, 599 (1993).

${ }^{5}$ Q. Peng, Y. P. Yi, Z. G. Shuai, and J. S. Shao, J. Am. Chem. Soc. 129, 9333 (2007).

${ }^{6}$ H. Wang and M. Thoss, J. Phys. Chem. A 111, 10369 (2007).

7 Y. Zhao and W. Z. Liang, Chem. Soc. Rev 41, 1075 (2012).

${ }^{8}$ H. Zang, Y. L. Ke, Y. Zhao, and W. Z. Liang, J. Phys. Chem. C 120, 13351 (2016).

9 Y. A. Yan, J. Chem. Phys. 150, 074106 (2019).

10 C. Hsieh and J. Cao, J. Chem. Phys. 148, 014104 (2018).

11 J. T. Hsiang and B. L. Hu, Phys. Rev. D 101, 125002 (2020).

12 J. T. Hsiang and B. L. Hu, Phys. Rev. D 101, 125003 (2020).

13 O. K. Rice and H. C. Ramsperger, J. Am. Chem. Soc. 49, 1617 (1927).

14 O. K. Rice and H. C. Ramsperger, J. Am. Chem. Soc. 50, 617 (1928).

15 L. S. Kassel, J. Phys. Chem. 32, 225 (1928).

16 L. S. Kassel, J. Phys. Chem. 32, 1065 (1928).

17 R. A. Marcus and O. K. Rice, J. Phys. Colloid. Chem. 55, 894 (1951).

18 R. A. Marcus, J. Chem. Phys. 20, 359 (1952).

19 K. A. Holbrook, M. Pilling, and S. Robertson, Unimolecular Reactions, Wiley, Chichester, UK, 1996, $2^{\text {nd }}$ ed.

20 M. G. Evans and M. Polanyi, Trans. Faraday Soc 31, 875 (1935).
21 H. Eyring, J. Chem. Phys. 3, 107 (1935)

22 H. Eyring, Chem. Rev. 17-1, 65 (1935).

23 C. N. Hinshelwood, The Kinetics of Chemical Change in Gaseous Systems, Clarendon Press, London, 1926.

24 Y. J. Yan, J. Chem. Phys. 140, 054105 (2014).

25 Y. J. Yan, J. S. Jin, R. X. Xu, and X. Zheng, Frontiers Phys. 11, 110306 (2016).

${ }^{26}$ H. D. Zhang, R. X. Xu, X. Zheng, and Y. J. Yan, Mol. Phys. 116, 780 (2018), Special Issue, "Molecular Physics in China".

27 Y. Wang, R. X. Xu, and Y. J. Yan, J. Chem. Phys. 152, $041102(2020)$.

28 R. X. Xu, Y. Liu, H. D. Zhang, and Y. J. Yan, Chin. J. Chem. Phys. 30, 395 (2017).

29 R. X. Xu, Y. Liu, H. D. Zhang, and Y. J. Yan, J. Chem. Phys. 148, 114103 (2018).

30 Y. Liu, R. X. Xu, H. D. Zhang, and Y. J. Yan, Chin. J. Chem. Phys. 31, 245 (2018).

31 U. Weiss, Quantum Dissipative Systems, World Scientific, Singapore, 2012, $4^{\text {rd }}$ ed.

${ }^{32}$ Y. J. Yan and R. X. Xu, Annu. Rev. Phys. Chem. 56, 187 (2005).

33 G. F. Bertsch, Online note: "Derivations of Marcus's formula".

34 L. Landau, Phys. Z. Sowjetunion 2, 46 (1932).

35 C. Zener, Proc. Roy. Soc. London A 137-6, 696 (1932).

36 C. Wittig, J. Phys. Chem. B 109, 8428 (2005).

37 A. O. Caldeira and A. J. Leggett, Physica A 121, 587 (1983).

38 C. Zhu, J. Chem. Phys. 105, 4159 (1996). 